\title{
Phase II trial of concurrent chemoradiotherapy with L-asparaginase and MIDLE chemotherapy for newly diagnosed stage I/II extranodal NK/T-cell Iymphoma, nasal type (CISL- 1008)
}

\author{
Dok Hyun Yoon ${ }^{1, *}$, Seok Jin Kim ${ }^{2, *}$, Seong Hyun Jeong ${ }^{3}$, Dong-Yeop Shin ${ }^{4}$, Sung \\ Hwa Bae ${ }^{5}$, Junshik Hong ${ }^{6}$, Seong Kyu Park7, Ho-Young Yhim ${ }^{8}$, Deok-Hwan Yang ${ }^{9}$, \\ Hyewon Lee ${ }^{10}$, Hye Jin Kang ${ }^{11}$, Mark Hong Lee ${ }^{12}$, Hyeon-Seok Eom ${ }^{10}$, Jae-Yong \\ Kwak ${ }^{8}$, Jae Hoon Lee ${ }^{6}$, Cheolwon Suh ${ }^{1}$ and Won Seog Kim² \\ ${ }^{1}$ Department of Oncology, Asan Medical Center, University of Ulsan College of Medicine, Seoul, Korea \\ 2 Department of Medicine, Samsung Medical Center, Sungkyunkwan University School of Medicine, Seoul, Korea \\ ${ }^{3}$ Department of Hematology-Oncology, Ajou University School of Medicine, Suwon, Korea \\ ${ }^{4}$ Department of Internal Medicine, Seoul National University Hospital, Seoul, Korea \\ ${ }^{5}$ Department of Internal Medicine, Catholic University of Daegu School of Medicine, Daegu, Korea \\ ${ }^{6}$ Department of Internal Medicine, Gachon University Gil Medical Center, Incheon, Korea \\ 7 Department of Hematology/Oncology, Soonchunhyang University Bucheon Hospital, Bucheon, Korea \\ ${ }^{8}$ Department of Internal Medicine, Chonbuk National University School of Medicine, Jeonju, Korea \\ ${ }^{9}$ Department of Internal Medicine, Chonnam National University Medical School, Gwangju, Korea \\ ${ }^{10}$ Center for Hematologic Malignancies, National Cancer Center, Goyang, Korea \\ ${ }^{11}$ Department of Internal Medicine, Korea Cancer Center Hospital, Korea Institute of Radiological and Medical Sciences, \\ Seoul, Korea \\ ${ }^{12}$ Department of Hematology-Oncology, Konkuk University Medical Center, Seoul, Korea \\ "These authors have contributed equally to this work \\ Correspondence to: Won Seog Kim, email: wskimsmc@skku.edu
}

Keywords: extranodal NK/T-cell lymphoma, nasal type; concurrent chemoradiotherapy; L-asparaginase; methotrexate; treatment Received: May15, 2016

Accepted: August 11, 2016

Published: August 16, 2016

\section{ABSTRACT}

We designed a new treatment protocol incorporating concurrent administration of L-asparaginase (to reduce the probability of systemic progression during concurrent chemoradiotherapy (CCRT)) plus high-dose methotrexate to consolidation chemotherapy to intensify the regimen for treating localized extranodal NK/T cell lymphoma, nasal type (ENKTL). CCRT comprised radiation (36-44 Gy) with weekly cisplatin ( $30 \mathrm{mg} / \mathrm{m}^{2}$ ) and tri-weekly L-asparaginase (4 $\left.000 \mathrm{IU}\right)$. ChemotherapyMIDLE (methotrexate $3 \mathrm{~g} / \mathrm{m}^{2}$ on day 1 , etoposide $100 \mathrm{mg} / \mathrm{m}^{2}$ and Ifosfamide 1000 $\mathrm{mg} / \mathrm{m}^{2}$ on days 2-3, dexamethasone $40 \mathrm{mg}$ on days 1-4, and L-asparaginase 6000 $\mathrm{IU} / \mathrm{m}^{2}$ on days $4,6,8,10$ )-was repeated every 28 days for two cycles. One of the 28 patients developed distant lesions after CCRT. The final complete response rate was $\mathbf{8 2 . 1 \%}$. Four patients dropped out during or after their first MIDLE cycle due to toxicities (recurrent G3 hyperbilirubinemia [ $=1$ ], G3-5 increased creatinine [n = 2], and G5 infection [ $=1]$ ). With a median follow-up of 46 months (95\% CI: 39-47 months), the estimated 3-year progression-free survival rate and overall survival rate were $\mathbf{7 4 . 1} \%$ and $\mathbf{8 1 . 5 \%}$, respectively. This MIDLE protocol may be effective for localized ENKTL. However, concurrent administration of L-asparaginase during CCRT does not seem to provide additional benefits. 


\section{INTRODUCTION}

Extranodal natural killer (NK)/T cell lymphoma (ENKTL) is a rare subtype of non-Hodgkin lymphoma (NHL) with a poor prognosis [1]. As the majority of ENKTL cases present as localized disease, particularly involving the nasal or paranasal area, treatment of localized disease has been an important issue $[1,2]$.

A Japanese group and our group have shown that concurrent chemoradiotherapy (CCRT) is a feasible and effective treatment for the management of localized ENKTL [2-4]. Furthermore, we have shown that CCRT with weekly cisplatin, followed by chemotherapy comprising etoposide, ifosfamide, cisplatin, and dexamethasone (VIPD) or etoposide, ifosfamide, dexamethasone, and L-asparaginase (VIDL) is an effective treatment strategy for the management of localized ENKTL $[2,3]$. All the studies resulted in promising outcomes with $70-80 \%$ overall survival. Although prognosis in these patients has improved in recent years, the failure pattern shows that systemic failure predominates and disease progression occurs early in the majority [5]. Two patients (6.7\%) among those enrolled in the phase 2 trial of VIDL also experienced systemic progression during CCRT, with development of new lesions in the liver or lung [2].

To further improve efficacy, we designed a new treatment protocol: methotrexate, ifosfamide, dexamethasone, L-asparaginase, and etoposide (MIDLE), which incorporates the tri-weekly administration of L-asparaginase (a key therapeutic agent for ENKTL) during CCRT to reduce the probability of systemic progression as well as high-dose methotrexate to intensify chemotherapy based on previous excellent outcomes of methotrexate-containing regimens such as dexamethasone, methotrexate, ifosfamide, L-asparaginase, etoposide (SMILE) and ifosfamide, methotrexate, L-asparaginase, prednisolone (IMEP) $[6,7]$.

\section{RESULTS}

\section{Patient characteristics}

Thirty patients with stage IE/IIE ENKTL were included this study from October 2010 to March 2012. Of these, two were excluded because of ineligibility ( $\mathrm{n}$ $=1)$ and refusal $(\mathrm{n}=1)$. The remaining 28 patients were included in the analysis. The median age at diagnosis was 51 years (range, $30-77$ years). Circulating EBV DNA was detected in 4 patients (median $1.295 \times 10^{6}$ copies $/ \mu \mathrm{L}$; range, $\left.655-4.6 \times 10^{6} \mathrm{copies} / \mu \mathrm{L}\right)$, while it was less than the detectable level in 24 patients (Table 1). PINK-E scores were 0 in 16 patients and 1 in 12 patients.

\section{Toxicity and response to CCRT}

All but two patients completed CCRT without interruption during treatment according to the protocol. One patient failed to finish CCRT following the protocol due to G3 mucositis with delayed recovery, and the other patient withdrew after G3 allergic reaction to L-asparaginase (Table 2). Three other patients stopped the administration of L-asparaginase during CCRT because of the development of repeated grade 2 allergic reactions to L-asparaginase, which was also not administered during MIDLE chemotherapy in those cases. Another two patients skipped their doses of cisplatin and L-asparaginase

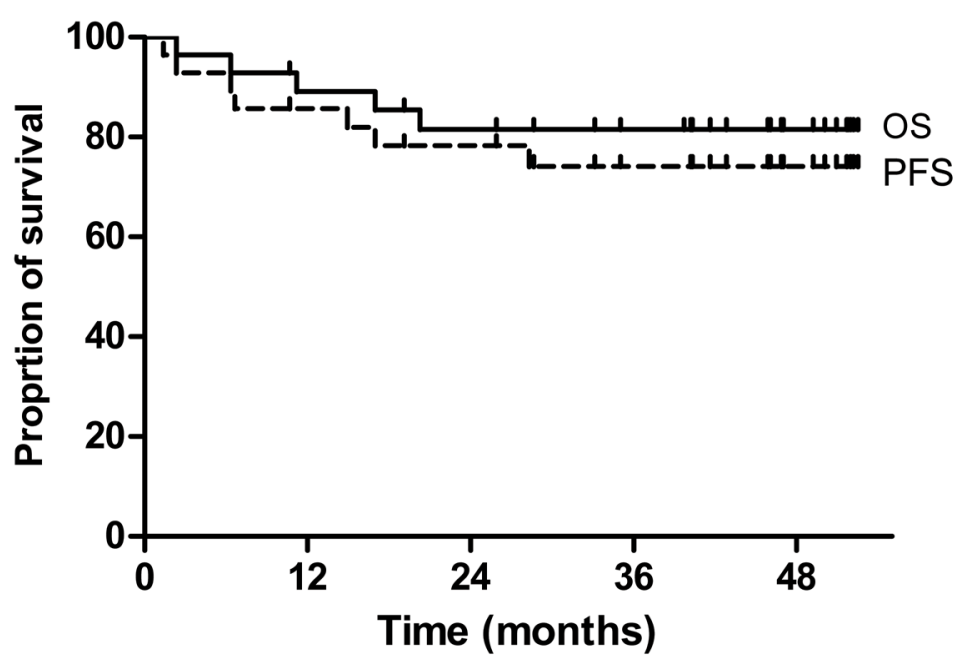

Figure 1: Progression-free survival (PFS) and overall survival (OS). 
Table 1: Sociodemographic and clinical characteristics of the studied patients

\begin{tabular}{|l|l|l|l|}
\hline & & \multicolumn{2}{c|}{ Total (n= 28) } \\
\hline \multicolumn{1}{|c|}{ Characteristics } & \multicolumn{2}{|c|}{ Number } & \% \\
\hline Age & Median 51, range: $30-77$ years & 7 & 25.0 \\
\hline & $>60$ & 24 & 85.7 \\
\hline Sex & Male & 28 & 100.0 \\
\hline Performance status & ECOG 0/1 & 22 & 78.6 \\
\hline Ann Arbor stage & IE & 6 & 21.4 \\
\hline & IIE & 3 & 10.7 \\
\hline Serum LDH & Increased & 4 & 14.3 \\
\hline B symptoms & Presence & 4 & 14.3 \\
\hline EBV in blood & Presence & 5 & 17.9 \\
\hline $\begin{array}{l}\text { Regional lymph node } \\
\text { involvement }\end{array}$ & Presence & 25 & 89.3 \\
\hline Primary site & Nasal cavity & 16 & 10.7 \\
\hline & Nasopharynx or oropharynx & 3 & 57.1 \\
\hline PINK-E & 0 & 12 & 42.9 \\
\hline & 1 & &
\end{tabular}

Abbreviations: EBV, Epstein-Barr virus; IPI, international prognostic index; NKPI, NK/T cell lymphoma prognostic index; PINK-E, prognostic index for NK/T cell lymphoma-EBV.

Table 2: Toxicity profiles

\begin{tabular}{|c|c|c|c|c|c|c|c|c|}
\hline \multirow[t]{2}{*}{ Toxicity } & \multicolumn{4}{|c|}{ CCRT (n = 28) } & \multicolumn{4}{|c|}{ MIDLE $(n=23)$} \\
\hline & G1 & G2 & G3 & G4 & G1 & G2 & G3 & G4 \\
\hline \multicolumn{9}{|l|}{ Hematologic } \\
\hline Anemia & 3 & 2 & & & 2 & 3 & 2 & \\
\hline Neutropenia & & 1 & 2 & & & & 2 & 19 \\
\hline Thrombocytopenia & & & & & & 3 & 1 & 2 \\
\hline Febrile neutropenia & & & & & 1 & 1 & 10 & \\
\hline \multicolumn{9}{|l|}{ Non-hematologic } \\
\hline Nausea & 4 & 3 & 11 & & 4 & 2 & 6 & \\
\hline Vomiting & 5 & 3 & 3 & & 3 & 2 & & \\
\hline Diarrhea & 1 & 1 & & & 1 & 3 & & \\
\hline Anorexia & 2 & 1 & 5 & & 6 & 3 & 4 & \\
\hline Constipation & 5 & 1 & & & 2 & 1 & & \\
\hline Stomatitis & 4 & 8 & 1 & & 6 & 6 & 2 & \\
\hline General weakness & 2 & & 1 & & 3 & 1 & 1 & \\
\hline Insomnia & 1 & 1 & & & 1 & 1 & & \\
\hline Allergic reaction & 2 & 3 & 1 & & 2 & 2 & 2 & \\
\hline Alopecia & 3 & & & & 3 & 3 & & \\
\hline Infection & & & & & & & & $1(\mathrm{G} 5)^{*}$ \\
\hline Creatinine elevation & & 2 & & & 1 & 1 & 1 & $1(\mathrm{G} 5)^{*}$ \\
\hline Amylase elevation & 2 & & & & & & & \\
\hline Transaminase elevation & 6 & 6 & 1 & & 2 & 3 & 3 & \\
\hline Bilirubin elevation & 1 & 8 & 3 & & 1 & 3 & 1 & \\
\hline
\end{tabular}

*A 51-year-old man died of acute kidney injury complicated by pneumonia with sepsis on C1D19 after MIDLE chemotherapy. 
Table 3: Regimens used for localized extranodal NK/T cell lymphoma

\begin{tabular}{|c|c|c|c|c|c|}
\hline & MIDLE $(n=28)$ & VIPD $(n=30)[3]$ & VIDL $(n=30)[2]$ & $\operatorname{DeVIC}(n=27)[4]$ & SMILE $^{\star}(n=38)[6]$ \\
\hline \multirow[t]{2}{*}{ Radiotherapy } & 36-44 Gy & $40-52.8 \mathrm{~Gy}$ & $40-44 \mathrm{~Gy}$ & $50 \mathrm{~Gy}$ & - \\
\hline & Weekly cisplatin $30 \mathrm{mg} / \mathrm{m}^{2}$ & & & & \\
\hline \multirow[t]{2}{*}{ Regimens for CCRT } & + triweekly L-asp 4,000 & Weekly cisplatin 30 mg/m² & Weekly cisplatin $30 \mathrm{mg} / \mathrm{m}^{2}$ & DeVIC & - \\
\hline & $\mathrm{IU} / \mathrm{m}^{2}$ & & & & \\
\hline Chemotherapy & & & & & - \\
\hline Methotrexate & $3 \mathrm{~g} / \mathrm{m}^{2}$ on $\mathrm{D} 1$ & - & - & - & $2 \mathrm{~g} / \mathrm{m}^{2}$ on $\mathrm{D} 1$ \\
\hline Epotoside & $100 \mathrm{mg} / \mathrm{m}^{2}$ on $\mathrm{D} 2-3$ & $100 \mathrm{mg} / \mathrm{m}^{2}$ on $\mathrm{D} 1-3$ & $100 \mathrm{mg} / \mathrm{m}^{2}$ on $\mathrm{D} 1-3$ & $67 \mathrm{mg} / \mathrm{m}^{2}$ on $\mathrm{D} 1-3$ & $100 \mathrm{mg} / \mathrm{m}^{2}$ on $\mathrm{D} 2-4$ \\
\hline Ifosfamide & $1,000 \mathrm{mg} / \mathrm{m}^{2}$ on $\mathrm{D} 2-3$ & $1,200 \mathrm{mg} / \mathrm{m}^{2}$ on $\mathrm{D} 1-3$ & $1,200 \mathrm{mg} / \mathrm{m}^{2}$ on $\mathrm{D} 1-3$ & $1,000 \mathrm{mg} / \mathrm{m}^{2}$ on $\mathrm{D} 1-3$ & $1,500 \mathrm{mg} / \mathrm{m}^{2}$ on $\mathrm{D} 2-4$ \\
\hline \multirow[b]{2}{*}{ Platinum } & \multirow[b]{2}{*}{-} & Cisplatin & \multirow[b]{2}{*}{ - } & Carboplatin & \multirow[b]{2}{*}{-} \\
\hline & & $33 \mathrm{mg} / \mathrm{m}^{2}$ on D1-3 & & $200 \mathrm{mg} / \mathrm{m} 2$ on D1 & \\
\hline Dexamethasone & $40 \mathrm{mg} /$ day on D1-4 & $40 \mathrm{mg} /$ day on D1-4 & $40 \mathrm{mg} /$ day on D1-4 & $40 \mathrm{mg} /$ day on D1-3 & $40 \mathrm{mg} /$ day on $\mathrm{D} 2-4$ \\
\hline L-asparaginase & $6,000 \mathrm{IU} / \mathrm{m}^{2}, 4$ doses & & $4,000 \mathrm{IU} / \mathrm{m}^{2}, 7$ doses & & $6,000 \mathrm{IU} / \mathrm{m}^{2}, 7$ doses \\
\hline \multirow{2}{*}{ No of cycles } & 2 cycles & 3 cycles & 2 cycles & 3 cycles & 2 cycles \\
\hline & every 4 weeks & every 3 weeks & every 4 weeks & every 3 weeks & Every 28 days \\
\hline CR rate & $82.1 \%$ & $80.0 \%$ & $87 \%$ & $77 \%$ & $45 \%^{*}$ \\
\hline PFS & 3yr PFS, $74.1 \%$ & 3yr PFS, $85.2 \%$ & 5yr PFS, $73 \%$ & 2yr PFS, $67 \%$ & 1yr PFS, 53\% \\
\hline OS & $3 y r$ OS, $81.5 \%$ & $3 y r$ OS, $86.3 \%$ & $5 y r$ OS, $60 \%$ & $2 \mathrm{yr}$ OS, $78 \%$ & $1 \mathrm{yr}$ OS, $55 \%$ \\
\hline G3-4 neutropenia & $91.3 \%$ & $46.7 \%$ & $80 \%$ & $90.9 \%$ & $100 \%$ \\
\hline G3-4 FN & $43.5 \%$ & $60 \%$ & $16.7 \%$ & $18.2 \%$ & NA \\
\hline TRM & $1 ; \mathrm{AKI}$ and pneumonia & 2; infection & 0 & 0 & 2 ; infection \\
\hline
\end{tabular}

*Phase 2 trial of SMILE involved stage 4, relapsed or refractory extranodal NK/T cell lymphoma unlike the other trials involving localized stage disease.

Abbreviations: AKI, acute kidney injury; CCRT, concurrent chemoradiotherapy; CR, complete response; D1, day 1. FN, febrile neutropenia; NA, not available; OS, overall survival; PFS, progression-free survival; TRM, treatment-related mortality.

due to grade 3 nausea/vomiting and grade 2 azotemia, respectively.

Sixteen patients (57.1\%) achieved CR and 8 a partial response (PR), with an overall response rate of $85.7 \%$. One patient showed stable disease (SD) after CCRT. The other patient showed progressive disease (PD) with development of new lesions in the retroperitoneal lymph node and spleen. This patient had the highest level of EBV $\left(4.6 \times 10^{6}\right.$ copies $\left./ \mu \mathrm{L}\right)$ at baseline. After completion of CCRT, two patients in CR after CCRT could not proceed to MIDLE chemotherapy treatment due to poor performance $(n=1)$ and decreased renal function $(n=1)$.

\section{Toxicity and response to MIDLE chemotherapy}

Twenty-three patients received MIDLE chemotherapy and 20 of those completed the planned 2 cycles of chemotherapy. However, MIDLE chemotherapy was associated with high rate of grade 3 or 4 neutropenia $(91.3 \%, \mathrm{n}=21)$ and febrile neutropenia $(43.5 \%, \mathrm{n}=10)$ (Table 2). Two patients, each on C1D2 (G3) and C1D3
(G5), experienced acute kidney injury and they could not proceed to the second cycle. One of them died of pneumonia complicated by sepsis after development of acute kidney injury. Another one was dropped from this study due to recurrent grade 3 bilirubinemia during cycle 1 . One patient required $25 \%$ dose reduction of methotrexate and skipped two doses of L-asparaginase during cycle 2 due to G3 mucositis. However, no patient had L-asparaginase-associated acute pancreatitis or coagulopathy during or after MIDLE chemotherapy. Administration of L-asparaginase was stopped in 2 patients due to grade 3 allergic reactions.

All 20 patients those who completed the 2 cycles of MIDLE chemotherapy achieved CR including those 6 patients with PR and 1 SD after CCRT. Therefore, the final CR rate was $82.1 \%$ (23 out of 28) after CCRT, followed by MIDLE chemotherapy. 


\section{Survival outcomes and prognostic indices}

The last survival data update was performed in July 2015. With a median follow-up of 46 months (95\% confidence interval: $39-47$ months), four patients progressed and five patients died with the estimated 3-year PFS rate of $74.1 \%$ and OS rate of $81.5 \%$, respectively (Figure 1).

A 50-year old man with detectable EBV in blood experienced local recurrence in the nasal cavity at 19.5 months and underwent retrial of CCRT followed by SMILE chemotherapy and high-dose chemotherapy with autologous stem cell transplantation (HDT-ASCT). The other three patients developed systemic progressive diseases. All of them were young ( $<60$ years), and EBV was detected in two of them at diagnosis. In other words, 3 of 4 patients (with EBV positivity in blood) experienced disease progression in contrast to only 1 of 24 with EBV negativity. One of the patients (who had disease progression at 28 months) was salvaged with SMILE and HDT-ASCT and survived, but the other two with disease progression just after CCRT (PFS 1.4 months) or MIDLE (PFS 6.4 months) died despite receiving salvage chemotherapy such as SMILE or IMEP. In addition to the two deaths due to PD, a 51-year old man died of pneumonia with sepsis complicated by acute kidney injury during MIDLE chemotherapy (as described above) and two additional elderly patients in CR (aged 72 and 77 years, respectively) died of pneumonia during followup; one died at 1 year after MIDLE chemotherapy and the other after 3.5 months.

Patients with a zero PINK-E score tended to have better PFS (3-year PFS rate of $87.5 \%$ vs. $54.0 \%, \mathrm{p}=$ 0.078 ) and OS (3-year OS rate of $87.5 \%$ vs. $73.3 \%, p=$ 0.414 ) although those observations were not statistically significant (Supplementary Figure 1).

\section{DISCUSSION}

The aim of this trial was to develop a more effective treatment strategy with the incorporation of L-asparaginase in addition to weekly cisplatin during CCRT (to lower the risk of early systemic treatment failure) along with the addition of high-dose methotrexate to the VIDLlike chemotherapy with modifications including reduced dose of both etoposide and ifosfamide [2]. This treatment resulted in a $82.1 \%$ CR rate with an estimated 3-year PFS rate of $74.1 \%$ and OS rate of $81.5 \%$.

All patients in PR or SD after CCRT could achieve CR after two cycles of MIDLE chemotherapy. Efficacies seem comparable to those from prior trials of VIPD, VIDL, and DeVIC; CR rates ranging from $77 \%$ to $87 \%$, 2 - to 5 -year PFS rates $67 \%$ to $85.2 \%$, and 2- to 5 -year OS rates $60 \%$ to $86.3 \%$ (Table 3 ) [2-4].

Although we aimed to reduce the probability of systemic failure during CCRT, one patient (3.6\%) developed new lesions in the distant lymph node and spleen just after CCRT. Concurrent administration of L-asparaginase during CCRT does not seem to provide additional benefit, although we cannot make definite conclusions comparing this failure rate with prior trials including VIPD ( 0 of 30) and VIDL ( 2 out of 30) which utilized just weekly cisplatin during CCRT. However, six patients experienced hypersensitivity to L-asparaginase during chemoradiotherapy resulting in skipping of doses or discontinuation of L-asparaginase. In addition, administration of L-asparaginase during MIDLE chemotherapy was stopped in two additional patients due to the allergic reactions.

Clinical hypersensitivity to native Escherichia coli L-asparaginase has been reported to range from $32.5 \%$ to $75 \%$ [8]. In the SMILE chemotherapy, 5 out of 38 patients experienced grade 1 to 3 allergic reactions to L-asparaginase, which was not observed during VIDL. Grade 3 or 4 gastrointestinal toxicity, including nausea (n $=11)$, vomiting $(\mathrm{n}=3)$, anorexia $(\mathrm{n}=5)$, and cholestatic injury with hyperbilirubinemia $(\mathrm{n}=3)$, was also relatively common compared with prior studies during CCRT. Thus, L-asparaginase should be administered with caution and vigilant monitoring of possible adverse events is advised during concurrent chemoradiotherapy.

Although the frequency of hematologic toxicitiesincluding grade 3 or 4 neutropenia $(n=21,91.3 \%)$ and febrile neutropenia $(\mathrm{n}=10,43.5 \%)$ - does not seem to be higher compared with those of prior trials (G3 or 4 neutropenia: $46.7 \%-91.3 \%$; G3 or 4 febrile neutropenia: $16.7 \%-60 \%$; Table 3); those rates seem to be still significantly high considering the generally fair prognosis of the localized ENKTL [2-4]. In addition, two patients experienced acute kidney injury on day 2 or 3 of the first cycle of MIDLE and one of them died of pneumonia complicated by sepsis, which seems to be related to highdose methotrexate.

Nephrotoxicity is also a significant toxicity in SMILE (containing $2.0 \mathrm{~g} / \mathrm{m}^{2}$ methotrexate); $5 \%$ experienced grade 3 azotemia in the phase 2 trial and $17.2 \%$ (including one acute kidney injury and death) experienced grade 3 azotemia in a study from the Asia Lymphoma Study Group [6, 9]. These risks of renal failure in patients with ENKTL seem too high compared with the $2 \%$ recorded in children treated with high-dose methotrexate for osteosarcoma [10]. Precautions with strict prophylactic measures including alkaline hydration must therefore be taken with such regimens containing high-dose methotrexate.

Recently we proposed both PINK and PINK-E as prognostic models of ENKTL in nonanthracycline-based treatment [11]. However, PINK must have a limitation in discriminating prognosis in this early-stage nasaltype disease because all the patients have stage 1 or 2 disease, nasal involvement, and no distant lymph node 
involvement therefore leaving just age as a valid variable for this model. In the case of PINK-E, both age and EBVPCR would be effective predictors among five variables resulting in classification of elderly patients with EBVPCR positivity as an intermediate-risk group. Although no enrolled patients were classified as higher-risk due to their small number, those with PINK-E score 1 still had a tendency for lower PFS and OS rates compared with those with PINK-E score 0.

In conclusion, L-asparaginase plus CCRT followed by MIDLE chemotherapy may be an effective treatment strategy for stage I/II extranodal NK/T-Cell lymphoma, nasal type. However, compared with a previous study, higher numbers of patients were discontinued during or after CCRT due to toxicity or poor tolerance. Concurrent administration of E. coli L-asparaginase during CCRT does not seem to provide additional benefits. Precautions should be taken when a high-dose methotrexate-containing regimen is given for early-stage disease due to possible adverse effects.

\section{MATERIALS AND METHODS}

\section{Patients}

Eligibility criteria included newly diagnosed ENKTL based on the presence of histological features and immunohistochemistry results, including cytoplasmic $\mathrm{CD} 3+, \mathrm{CD} 20-$, and CD56+ positive for cytotoxic molecules and Epstein-Barr virus (EBV) in in situ hybridization. None of the patients received any treatment for ENKTL, and all had measurable disease.

Patients were 18 years of age or older, and their disease state was Ann Arbor stage IE or IIE. Additional eligibility criteria were as follows: Eastern Cooperative Oncology Group (ECOG) performance status of 0-2; hemoglobin $\geq 9.0 \mathrm{~g} / \mathrm{dL}$; absolute neutrophil count $\geq 1500 /$ $\mu \mathrm{L}$ and platelet count $\geq 100000 / \mu \mathrm{L}$; serum creatinine levels $\leq 1.5 \mathrm{mg} / \mathrm{dL}$ and creatinine clearance $\geq 50 \mathrm{~mL} / \mathrm{min}$; total bilirubin < two times the upper limit of normal; and aspartate transferase $<$ three times the upper limit of the normal value.

Patients who had any coexisting medical diseases with sufficient severity to prevent full compliance with the study protocol such as heart failure or acute, active infection were excluded from the study. Considering the adverse effects of L-asparaginase, patients who had a history of acute pancreatitis were also excluded.

\section{Study design and objectives}

CCRT consisted of radiation therapy with 36-44 Gy per 18-22 fractions and weekly administration of cisplatin $\left(30 \mathrm{mg} / \mathrm{m}^{2}\right)$ and tri-weekly intravenous or intramuscular
L-asparaginase (4 $000 \mathrm{IU} / \mathrm{m}^{2}$ ) on days 1,3 , and 5 in each week during radiotherapy. All patients received three-dimensional conformal radiotherapy or intensitymodulated radiotherapy. The clinical target volume was delineated with adequate margins surrounding the gross target volume, considering the anatomic boundary of the involved subsite. The addition of elective lymphatic irradiation was determined on an individual basis $[3,12]$. The first response evaluation was performed 3 to 4 weeks after the completion of CCRT.

Two cycles of MIDLE chemotherapy (methotrexate $3 \mathrm{~g} / \mathrm{m}^{2}$ on day 1, intravenous administration of etoposide $\left(100 \mathrm{mg} / \mathrm{m}^{2}\right)$, ifosfamide $\left(1000 \mathrm{mg} / \mathrm{m}^{2}\right)$, and dexamethasone (40 mg) on days $2-4$, followed by intravenous or intramuscular injection of L-asparaginase (4 $000 \mathrm{IU} / \mathrm{m}^{2}$ on days $4,6,8$, and 10 ) was repeated every 4 weeks. The final response evaluation was performed 6 to 8 weeks after the completion of the second cycle of MIDLE. The primary endpoint was complete response (CR) rate, including CR-unconfirmed (CR-u) by investigator review; and the secondary objectives included overall response rate, overall survival (OS), progression-free survival (PFS), and toxicities.

All patients provided written informed consent. The study was reviewed and approved by the institutional review board at each participating institute and was registered at ClinicalTrials.gov (NCT01238159).

\section{Assessment of response and toxicity}

Baseline assessments included complete blood count, determination of serum lactate dehydrogenase (LDH) levels, bone marrow aspiration and trephine biopsy, endoscopic examination of the nasal and oral cavities by otorhinolaryngologists, CT scanning or magnetic resonance imaging (MRI) of the involved lesions, CT scanning of the chest and abdomen-pelvis, and ${ }^{18} \mathrm{~F}$-fluorodeoxyglucose positron emission tomography (FDG-PET).

All these studies were performed before treatment and after completion of CCRT and MIDLE, and, to monitor relapse, they (except FDG-PET) were then repeated every 6 months thereafter for 2 years and yearly thereafter for the following 3 years. Subsequently, survival status was monitored at each participating institute, and evaluation of disease status to monitor relapse was performed at the physicians' discretion.

For prognostic factor analysis, prognostic index for NK/T cell lymphoma-EBV (PINK-E) was evaluated [11]. Quantitative polymerase chain reaction of EBV DNA in the peripheral blood was also performed as proposed previously $[13,14]$. The treatment response was assessed according to the International Working Group response criteria [15]. Toxicity was evaluated before each treatment cycle according to the National Cancer Institute Common Toxicity Criteria (NCI CTC) version 4.0. Dose 
modification of cisplatin during CCRT as well as that of etoposide and ifosfamide was performed as reported previously [3].

If patients experienced grade 1 or 2 allergic reactions or hypersensitivity to L-asparaginase, treatment was interrupted until the toxicity resolved and treatment was resumed with a $50 \%$ dose reduction; however, it was omitted if the toxicity repeated. If the patients had grade 3 or 4 allergic reactions or hypersensitivity, pancreatitis, or hypotension, then L-asparaginase was omitted.

\section{Statistics}

The sample size was determined based on CR rates according to Simon's optimal two-stage design [17] and assuming a target and a lower activity level of 0.90 (p1) and $0.70(\mathrm{p} 0)$, respectively. The minimum target number of subjects was 27 in total. This design provided a probability of 0.05 of accepting a treatment worse than $\mathrm{p} 0$ and a probability of 0.20 for rejecting a treatment better than $\mathrm{p} 1$. If we assume that the dropout rate is $10 \%$, the total accrual needed to be 30 patients.

The association between patient characteristics and treatment response was analyzed using the Chi-square test. The Kaplan-Meier method was used to calculate PFS and OS, and survival curves were compared via the log-rank test. A two-sided $\mathrm{P}$ value $<0.05$ was considered significant. PFS was defined as the time from the date of enrollment to the date of documented disease progression or any kind of death. OS was measured from the date of enrollment to the date of death from any cause and was censored at the date of the last follow-up visit.

\section{ACKNOWLEDGMENTS}

We thank the patients and their families as well as all the investigators, including the physicians and the coordinators in the CISL.

\section{CONFLICTS OF INTEREST}

None of the authors have relevant conflicts of interest.

\section{REFERENCES}

1. Lee J, Suh C, Park YH, Ko YH, Bang SM, Lee JH, Lee DH, Huh J, Oh SY, Kwon HC, Kim HJ, Lee SI, Kim JH, Park J, Oh SJ, Kim K, et al. Extranodal natural killer T-cell lymphoma, nasal-type: a prognostic model from a retrospective multicenter study. Journal of clinical oncology. 2006; 24(4):612-618.

2. Kim SJ, Yang DH, Kim JS, Kwak JY, Eom HS, Hong DS, Won JH, Lee JH, Yoon DH, Cho J, Nam TK, Lee SW, Ahn $\mathrm{YC}$, Suh $\mathrm{C}$ and Kim WS. Concurrent chemoradiotherapy followed by L-asparaginase-containing chemotherapy, VIDL, for localized nasal extranodal NK/T cell lymphoma: CISL08-01 phase II study. Annals of hematology. 2014; 93(11):1895-1901.

3. Kim SJ, Kim K, Kim BS, Kim CY, Suh C, Huh J, Lee SW, Kim JS, Cho J, Lee GW, Kang KM, Eom HS, Pyo HR, Ahn YC, Ko YH and Kim WS. Phase II trial of concurrent radiation and weekly cisplatin followed by VIPD chemotherapy in newly diagnosed, stage IE to IIE, nasal, extranodal NK/T-Cell Lymphoma: Consortium for Improving Survival of Lymphoma study. Journal of clinical oncology. 2009; 27(35):6027-6032.

4. Yamaguchi $M$, Tobinai $K$, Oguchi $M$, Ishizuka $N$, Kobayashi Y, Isobe Y, Ishizawa K, Maseki N, Itoh K, Usui N, Wasada I, Kinoshita T, Ohshima K, Matsuno Y, Terauchi T, Nawano S, et al. Phase I/II study of concurrent chemoradiotherapy for localized nasal natural killer/T-cell lymphoma: Japan Clinical Oncology Group Study JCOG0211. Journal of clinical oncology. 2009; 27(33):5594-5600.

5. Li YX, Liu QF, Wang WH, Jin J, Song YW, Wang SL, Liu YP, Liu XF, Zhou LQ and Yu ZH. Failure patterns and clinical implications in early stage nasal natural killer/T-cell lymphoma treated with primary radiotherapy. Cancer. 2011; 117(22):5203-5211.

6. Yamaguchi M, Kwong YL, Kim WS, Maeda Y, Hashimoto C, Suh C, Izutsu K, Ishida F, Isobe Y, Sueoka E, Suzumiya J, Kodama T, Kimura H, Hyo R, Nakamura S, Oshimi K, et al. Phase II study of SMILE chemotherapy for newly diagnosed stage IV, relapsed, or refractory extranodal natural killer (NK)/T-cell lymphoma, nasal type: the NK-Cell Tumor Study Group study. Journal of clinical oncology. 2011; 29(33):4410-4416.

7. Lee KW, Yun T, Kim DW, Im SA, Kim TY, Yoon SS, Heo DS, Bang YJ, Park S, Kim BK and Kim NK. Firstline ifosfamide, methotrexate, etoposide and prednisolone chemotherapy +/- radiotherapy is active in stage I/II extranodal NK/T-cell lymphoma. Leukemia \& lymphoma. 2006; 47(7):1274-1282.

8. Shinnick SE, Browning ML and Koontz SE. Managing hypersensitivity to asparaginase in pediatrics, adolescents, and young adults. Journal of pediatric oncology nursing. 2013; 30(2):63-77.

9. Kwong YL, Kim WS, Lim ST, Kim SJ, Tang T, Tse E, Leung AY and Chim CS. SMILE for natural killer/T-cell lymphoma: analysis of safety and efficacy from the Asia Lymphoma Study Group. Blood. 2012; 120(15):2973-2980.

10. Widemann BC, Balis FM, Kempf-Bielack B, Bielack S, Pratt CB, Ferrari S, Bacci G, Craft AW and Adamson PC. High-dose methotrexate-induced nephrotoxicity in patients with osteosarcoma. Cancer. 2004; 100(10):2222-2232.

11. Kim SJ, Yoon DH, Jaccard A, Chng WJ, Lim ST, Hong H, Park Y, Chang KM, Maeda Y, Ishida F, Shin DY, Kim JS, Jeong SH, Yang DH, Jo JC, Lee GW, et al. A prognostic index for natural killer cell lymphoma after non- 
anthracycline-based treatment: a multicentre, retrospective analysis. The Lancet Oncology. 2016; 17(3):389-400.

12. Oh D, Ahn YC, Kim SJ, Kim WS and Ko YH. Concurrent Chemoradiation Therapy Followed by Consolidation Chemotherapy for Localized Extranodal Natural Killer/TCell Lymphoma, Nasal Type. International journal of radiation oncology, biology, physics. 2015; 93(3):677-683.

13. Kim HS, Kim KH, Kim KH, Chang MH, Ji SH, Lim do H, Kim K, Kim SJ, Ko Y, Ki CS, Jo SJ, Lee JW and Kim WS. Whole blood Epstein-Barr virus DNA load as a diagnostic and prognostic surrogate: extranodal natural killer/T-cell lymphoma. Leukemia \& lymphoma. 2009; 50(5):757-763.

14. Kim SJ, Choi JY, Hyun SH, Ki CS, Oh D, Ahn YC, Ko YH, Choi S, Jung SH, Khong PL, Tang T, Yan X, Lim ST, Kwong YL and Kim WS. Risk stratification on the basis of Deauville score on PET-CT and the presence of Epstein-Barr virus DNA after completion of primary treatment for extranodal natural killer/T-cell lymphoma, nasal type: a multicentre, retrospective analysis. The Lancet Haematology. 2015; 2(2):e66-74.
15. Cheson BD, Horning SJ, Coiffier B, Shipp MA, Fisher RI, Connors JM, Lister TA, Vose J, Grillo-Lopez A, Hagenbeek A, Cabanillas F, Klippensten D, Hiddemann W, Castellino R, Harris NL, Armitage JO, et al. Report of an international workshop to standardize response criteria for non-Hodgkin's lymphomas. NCI Sponsored International Working Group. Journal of clinical oncology. 1999; 17(4):1244. 